\title{
Fungal Respiratory Infections in Cystic Fibrosis (CF): Recent Progress and Future Research Agenda
}

\author{
Jean-Philippe Bouchara • Françoise Symoens • Carsten Schwarz • \\ Vishnu Chaturvedi
}

Published online: 19 January 2018

(C) Springer Science+Business Media B.V., part of Springer Nature 2018

The World Health Organization estimates nearly $70,000-100,000$ worldwide cases of cystic fibrosis (CF) with a majority of diagnosis confirmed among the Caucasian populations in Europe and North America [1, 2]. Most CF patients suffer from respiratory infections due to Pseudomonas aeruginosa and other bacterial pathogens [3, 4]. Much less is known about the significance of fungal pathogens recovered from CF patients, the best methods to detect them and therefore the real frequency of fungal infections, or the pathogenesis of fungal airway colonization and

J.-P. Bouchara $(\bowtie) \cdot$ F. Symoens

Groupe d'Etude des Interactions Hôte-Pathogène (EA 3142), UNIV Angers, UNIV Brest, Université BretagneLoire, Institut de Biologie en Santé-IRIS, 4 rue Larrey, 49933 Angers, France

e-mail: jean-philippe.bouchara@univ-angers.fr

\section{J.-P. Bouchara}

Laboratoire de Parasitologie-Mycologie, Centre Hospitalier Universitaire, Institut de Biologie en SantéPBH, CHU, 4 rue Larrey, 49933 Angers, France

C. Schwarz

Department of Pediatric Pneumology and Immunology, Cystic Fibrosis Center Berlin, Charité-

Universitätsmedizin, Augustenburger Platz 1, 13353 Berlin, Germany

V. Chaturvedi $(\bowtie)$

California Institute for Medical Research, 2260 Clove

Drive, San Jose, CA 95128, USA

e-mail: eic@mycopathologia.net respiratory infections. This special issue of $\mathrm{Myco}$ pathologia brings together a large group of international experts to provide insightful articles on the different aspects of pulmonary fungal infections in $\mathrm{CF}$ patients. The work reflects the activities of the ECMM/ISHAM working group on Fungal respiratory infections in Cystic Fibrosis (Fri-CF).

The articles are organized into sections dealing with clinical aspects, laboratory diagnosis, treatment, ecology and pathogenesis (Table 1). In the first article, a team led by Sedlacek sketches the scope of the special issue with a comprehensive description of the evolving epidemiology and best management practices for the CF patients as per the standards of the European Cystic Fibrosis Society [5]. Their multicenter overview highlights the increasing involvement of the fungal pathogens and the critical role of the clinical microbiologists for providing tests tailored to the special needs of the CF patients. The authors review available evidence for allergenic and invasive diseases caused by the pathogenic molds and yeasts recovered from the respiratory specimens in nearly one-third of the CF patients [5]. Schwarz et al. [6] highlight the therapeutic strategies and preventative measurements for fungal infections in patients with CF. The authors recommend seven criteria for the diagnosis of "highly probable" fungal pneumonia in CF patients. A critical examination of available information is the basis for evaluation of the etiologic roles of Aspergillus species, Candida species, Lomentospora prolificans, and rare 
Table 1 Articles map for the special issue on respiratory fungal diseases in CF patients

\begin{tabular}{ll}
\hline Article subject & References \\
\hline 1. Fungal epidemiology in CF patients & {$[5,6]$} \\
2. Fungal diagnostic criteria in CF patients & {$[6,9,12,13]$} \\
3. Antifungal therapy in CF patients & {$[7,9,14-17]$} \\
4. Aspergillus, Exophiala and Pneumocystis in CF patients & {$[8-11]$} \\
5. Microbiome and ecology of fungal pathogens & {$[18-20]$} \\
6. Host response of fungal diseases in CF patients & {$[21]$} \\
7. Fungal virulence mechanisms & {$[22-27]$} \\
\hline
\end{tabular}

fungal pathogens. The efficacy of antifungals drugs, used as single antifungal treatment or in combinations, is detailed along with new therapies and prevention strategies [6]. The third article in the clinical section is from another group led by Schwarz [7]. It provides details of the clinical management for eighteen cases of respiratory mycoses from six different $\mathrm{CF}$ centers of excellence. The etiologic agents include Aspergillus species, Lomentospora prolificans, and other pathogenic fungi [7]. Many of the patients had clinical complications due to comorbidities, but good outcomes were obtained with the triple combination of antifungal drugs in nearly half of the cases. The article by Schwarz et al. [7] underscores the unmet needs for biomarkers to track fungal diseases and accurate antifungal laboratory tests to direct antifungal combination therapy.

The clinical section on individual pathogens includes two articles describing Aspergillus in CF bronchiectasis and Aspergillus bronchitis [8, 9]. Chotirmall and Martin-Gomez [8] summarize existing knowledge on Aspergillus colonization, allergic, chronic or invasive disease spectrum, and diagnostic and management challenges. The authors compare bronchiectasis in $\mathrm{CF}$ and non-CF patients and the presence of Aspergillus both as cause and consequence. Chotirmall and Martin-Gomez [8] have laid out a roadmap for future research on mycobiome, biomarkers, imaging and treatment modalities that would lead to better insight into Aspergillus in the setting of bronchiectasis. Schwarz et al. [9] report a retrospective analysis of patient data from the German CF Registry and prospective study of additional patients to summarize information on Aspergillus bronchitis, which is a well-defined clinical entity in CF. Their results show Aspergillus colonization in about $32.5 \%$ of 2599 patients, low prevalence of Aspergillus bronchitis (1.6-9\%) and relatively good outcomes with the targeted antifungal therapy [9]. Grenouillet et al. [10] focus on the higher recovery of some fungal species, as exemplified here with $E x$ ophiala dermatitidis, from the respiratory specimens of CF patients vis-à-vis non-CF patients. The two case presentations including long-term laboratory followup included culture recovery and intermittent antifungal treatment, and the demonstration of anti-E. dermatitidis IgG in one patient. These two case presentations indicated that the recovery of some fungi like $E$. dermatitidis in patients with bronchiectasis should lead to search for a mild form of CF [10]. Nevez et al. [11] detail the extensive laboratory processes setup in Brittany for the demonstration of Pneumocystis jirovecii in CF patients. The prevalence of Pneumocystis jirovecii was found to be $2.5 \%$ in Brittany despite the overall higher prevalence of $\mathrm{CF}$ in the population [11]. The authors also describe successful genotyping of $P$. jirovecii in two of the three instances to set the stage for future evaluation of links between genotype evolution and virulence [11].

Three articles cover the complex laboratory diagnosis of fungal respiratory diseases in CF patients. Chen et al. [12] outline the urgent need for standardization of laboratory methods to diagnose fungal colonization and infection in CF patients. The authors focus on both traditional culture and molecular methods. They provide critical examination of available data on innovations such as homogenized sputa and selective media for the recovery of fungi from respiratory specimens. Similarly, panfungal PCR, DNA sequencing, MLST and mass spectrometry methods are reviewed as the solid foundations for the diagnosis of fungal disease in CF [12]. Delhaes and the group from the "MucoFong" program summarize the results of systematic investigation on the optimal culture methods for recovery of fungal pathogens from the respiratory specimens of CF patients [13]. This 
large study involved $243 \mathrm{CF}$ patients from seven centers in France over a 15-month period. The investigators found that about half of the patients presented with at least one fungal species, while $37 \%$ were co-colonized with two species. The investigative team recommended a standardized protocol with the use of four culture media to facilitate the growth of Aspergillus species and yeasts, and slow-growing molds with a 16-day incubation period [13]. Hector et al. [14] also write critically about the unsolved issues around fungal colonization, sensitization or invasion of $\mathrm{CF}$ airways. Additionally, the authors provide an impressive schematic about the recognition of fungal pathogens by various receptors on the innate immune cells. Although the authors' main focus is on A. fumigatus, C. albicans, E. dermatitidis and $P$. jirovecii, they also examine the role of other fungi as well as bacteria from the culture-independent microbiome studies. The co-colonization of CF lungs by bacteria especially Pseudomonas aeruginosa and Staphylococcus aureus is evaluated for its likely impact on fungi in CF lungs [14].

The triazole antifungals are metabolized variably and sub-optimally in CF patients, and the resultant pharmacokinetics derangements could complicate treatment and even lead to the emergence of antifungal resistance. Rivosecchi et al. [15] expertly summarize limited pharmacokinetics data for fluconazole, itraconazole, voriconazole, isavuconazole and posaconazole for CF patients. They conclude by evidence-based recommendations on the benefits of therapeutic drug monitoring for azoles in $\mathrm{CF}$ patients. Azole resistance in A. fumigatus has been a major concern for patients with invasive aspergillosis, and this extends to $\mathrm{CF}$ patients. Hamprecht et al. [16] cover the epidemiology, diagnosis, treatment and environmental prevalence of azole-resistant A. fumigatus (ARAf). The authors provide insight into culture and sequencingbased assays for the detection and confirmation of ARAf. Hamprecht et al. [16] conclude with a brief description of the current treatment options for ARAf. Nagl et al. [17] describe the application of N-chlorotaurine (NCT) as a potent antiseptic and anti-inflammatory compound that could be delivered safety via inhalation. The broad antifungal activity of NCT in vitro and in the milieu of CF mucus and its impact on attenuation of virulence augur well for its clinical application. NCT safety profile has been confirmed with testing in pigs and mouse models as well as in the investigators themselves acting as human testers. A recently completed Phase I safety trial has not revealed any adverse effect in 24 human subjects although more extensive data analysis is pending [17].

Botterel et al. [18] describe the complexity of microbes in the airways of CF patients using both culture-dependent and culture-independent methods. Overall, a reduced fungal and bacterial diversity was revealed in the patients with severe airways disease. Four test patients harbored 27 bacterial and 18 fungal genera. Not unexpectedly, there was a discordance between the results obtained by the culture and sequencing methods [18]. This study established that the core mycobiota composed of Aspergillus belonging to the Fumigati section and Penicillium sp. The notable presence of Scedosporium species in the CF respiratory specimens is addressed in a broader ecological context by Rougeron et al. [19]. The authors review ecological niche of Scedosporium species in indoor and outdoor environment with $S$. apiospermum and S. boydii dominating both of these niches in France. Scedosporium species' ability to degrade aliphatic and aromatic pollutants and the impact of human activities on their ecological niches are evaluated in the context of their relative abundance in CF airways [19]. A similar theme could be found in the article by Zalar et al. [20] with the focus on $E$. dermatitidis colonization in CF patients. The pathogen has wide distribution in biosphere including the nutrient poor and polluted environments. Zalar et al. [20] present an informative schematic to summarize the diverse ecological niches occupied by $E$. dermatitidis and the possible source for human colonization.

The last section of the special issue comprises of seven articles on the pathogenesis and virulence of fungal pathogens. Scheffold et al. [21] begin the section by a brief review of current knowledge about the fungal pathogens in CF patients. They propose that protective or harmful $\mathrm{T}$ cell populations, specific to the respective fungal pathogens, could be the key to determining the exact etiologic roles played by fungi. As T-cell repertoire is quite complex with multiple subpopulations, the authors present a summary illustration of the host-fungus interactions reflected by the fungus-specific CD4+-T cells. The authors then describe the potential benefits of investigations that seek fungus-specific $\mathrm{T}$ cells as diagnostics tools in $\mathrm{CF}$ patients [21]. The group led by Fleury details detoxifying enzymes employed by the fungal pathogens in 
response to reactive-oxygen and -nitrogen species (ROS and RNS) released by the host phagocytic cells [22]. Scedosporium apiospermum genome sequence is the basis of cataloging the 33 genes likely to encode relevant detoxifying enzymes against host ROS and RNS. The domain organizations of superoxide dismutase and catalases genes are summarized [22]. The homologs of these and other related enzymes are also compared for $C$. albicans, $E$. dermatitidis and Aspergillus species. Staerck et al. [22] conclude with the description of development of specific immunodiagnostic tool based upon purified catalase of $S$. boydii [22]. The genetic manipulations of fungal pathogens have yielded valuable insights into their virulence mechanisms. This experimental approach is now possible with the disruption of $\mathrm{Cu} / \mathrm{Zn}$-superoxide dismutase (SOD) gene in S. aurantiacum [23]. The research group led by Sandrine Giraud used dominant selection marker for the construction of the SOD disruption mutants and confirmed increased susceptibility to oxidative stress by the menadione sensitivity test [23]. This proof-of-concept study is likely to lead to future in-depth experiments on the precise mechanisms of host-pathogen interactions in disease caused by $S$. aurantiacum and other Scedosporium species.

Pseudomonas aeruginosa is a significant pathogen in $\mathrm{CF}$ patients, and it is reported to influence the microbiome of respiratory system. Two research groups report inhibitory activities of $P$. aeruginosa on Scedosporium species, L. prolificans and A. fumigatus [24, 25]. Chen et al. [24] discovered that nonmucoid $P$. aeruginosa strain caused more inhibition of $S$. aurantiacum and L. prolificans in vitro. The fungal pathogens exhibited a loss of viability and the deformation of hyphal structures in response to $P$. aeruginosa; the effects were less pronounced with mucoid bacterial strains [24]. These exciting findings would allow future examination of the mechanism of colonization and infection caused by Scedosporium and Lomentospora in CF lungs where P. aeruginosa mucoid forms are predominant. Anand et al. [25] build upon the previous findings on the inhibition of $A$. fumigatus biofilms by non-mucoid $P$. aeruginosa by focusing on the role of small colony variants (SCV) of the bacterial pathogen on fungal inhibition. SCV $P$. aeruginosa isolates displayed heterogeneity in their inhibition of A. fumigatus biofilm vis-à-vis the nonmucoid bacterial strains. Further, $P$. aeruginosa pyoverdine production correlated with A. fumigatus inhibition [25]. Thus, the two complementary experimental studies have advanced our understanding of bacterial-fungal pathogens interactions likely to take place in CF patients.

The last two articles in the special issue describe innovative experimental approaches for the investigations of fungal pathogens in CF patients [26, 27]. The research group led by Rementeria uses proteomics approaches to investigate the cell wall and secretory proteins of Aspergillus species and Scedosporium/ Lomentospora species [26]. The authors use easy-tofollow schematics to outline research approaches and results obtained by proteomics-based approaches. Although all proteins are cataloged, there is special emphasis on the immunoreactive ones as they might have critical diagnostic and therapeutic applications in CF patients [26]. Cuénot and Bouchara [27] provide a fitting conclusion to this special issue by describing the exciting applications of atomic force microscopy (AFM) for understanding host-pathogen interactions in CF. The authors begin by providing a series of schematics about the use of AFM for the nanoscale resolution of cell surface structures and interactions under physiological conditions, which is not possible with other electron microscopy approaches. Next, they summarize key findings from the current and earlier studies using AFM images from Aspergillus species and Scedosporium species to highlight surface rodlet structures, germination changes and details of surface adhesion forces. The authors conclude by re-emphasizing the central role that AFM is likely to play in elucidation of cell surface interactions, molecular recognitions and innovative strategies to develop antiadhesion molecule to control fungal infections in $\mathrm{CF}$ patients [27].

The ECMM/ISHAM working group on Fungal respiratory infections in Cystic Fibrosis (Fri-CF) aimed this special issue of Mycopathologia to be both a showcase of the current progress and to suggest future research agenda. As with other scientific areas, many topics require more work for better understanding of the role of fungal pathogens in CF patients. Such expansion of knowledge would be crucial to ensure better management in increasingly older CF patient population with likely increase in the incidence of fungal diseases. It is sincerely hoped that the brief outlines presented in this editorial would encourage the reader to pursue respective articles in detail, to 
formulate their overall perspective of the research problems and to undertake creative research endeavors for the benefits of CF patients.

\section{References}

1. Mirtajani S, Farnia P, Hassanzad M, et al. Geographical distribution of cystic fibrosis; the past 70 years of data analyzis. Biomed Biotechnol Res J. 2017;1:105-12. https:// doi.org/10.4103/bbrj.bbrj_81_17.

2. O'Sullivan BP, Freedman SD. Cystic fibrosis. Lancet. 2009;373:1891-904. https://doi.org/10.1016/S01406736(09)60327-5.

3. Høiby N. Microbiology of lung infections in cystic fibrosis patients. Acta Paediatr Scand. 1982;Suppl 301:33-54. https://doi.org/10.1111/j.1651-2227.1982.tb09640.x.

4. Gilligan PH. Infections in patients with cystic fibrosis. Clin Lab Med. 2014;34:197-217. https://doi.org/10.1016/j.cll. 2014.02.001.

5. Schwarz C, Bouchara J-P, Buzina W, et al. Organization of patient management and fungal epidemiology in cystic fibrosis. Mycopathologia. 2017. https://doi.org/10.1007/ s11046-017-0205-x.

6. Schwarz C, Hartl D, Eickmeier O, et al. Progress in definition, prevention and treatment of fungal infections in cystic fibrosis. Mycopathologia. 2017. https://doi.org/10. 1007/s11046-017-0182-0.

7. Schwarz C, Brandt C, Whitaker P, et al. Invasive pulmonary fungal infections in cystic fibrosis. Mycopathologia. 2017. https://doi.org/10.1007/s11046-017-0199-4.

8. Chotirmall SH, Martin-Gomez MT. Aspergillus species in bronchiectasis: challenges in the cystic fibrosis and noncystic fibrosis airways. Mycopathologia. 2017. https://doi. org/10.1007/s11046-017-0143-7.

9. Brandt C, Roehmel J, Rickerts V, et al. Aspergillus bronchitis in patients with cystic fibrosis. Mycopathologia. 2017. https://doi.org/10.1007/s11046-017-0190-0.

10. Grenouillet F, Cimon B, Pana-Katatali H, et al. Exophiala dermatitidis revealing cystic fibrosis in adult patients with chronic pulmonary disease. Mycopathologia. 2017. https:// doi.org/10.1007/s11046-017-0218-5.

11. Nevez G, Robert-Gangneux F, Pougnet L, et al. Pneumocystis jirovecii and cystic fibrosis in Brittany, France. Mycopathologia. 2017. https://doi.org/10.1007/s11046017-0172-2.

12. Chen SC-A, Meyer W, Pashley CH. Challenges in laboratory detection of fungal pathogens in the airways of cystic fibrosis patients. Mycopathologia. 2017. https://doi.org/10. 1007/s11046-017-0150-8.

13. Coron N, Pihet M, Fréalle E, et al. Toward the standardization of mycological examination of sputum samples in cystic fibrosis: results from a French multicenter prospective study. Mycopathologia. 2017. https://doi.org/10.1007/ s11046-017-0173-1.

14. Singh A, Ralhan A, Schwarz C, Hartl D, Hector A. Fungal pathogens in $\mathrm{CF}$ airways: leave or treat? Mycopathologia. 2017. https://doi.org/10.1007/s11046-017-0184-y.
15. Rivosecchi RM, Samanta P, Demehin M, Nguyen MH. Pharmacokinetics of azole antifungals in cystic fibrosis. Mycopathologia. 2017. https://doi.org/10.1007/s11046017-0189-6.

16. Hamprecht A, Morio F, Bader O, et al. Azole resistance in Aspergillus fumigatus in patients with cystic fibrosis: a matter of concern? Mycopathologia. 2017. https://doi.org/ 10.1007/s11046-017-0162-4.

17. Nagl M, Arnitz R, Lackner M. N-Chlorotaurine, a promising future candidate for topical therapy of fungal infections. Mycopathologia. 2017. https://doi.org/10.1007/s11046017-0175-z.

18. Botterel F, Angebault C, Cabaret O, et al. Fungal and bacterial diversity of airway microbiota in adults with cystic fibrosis: concordance between conventional methods and ultra-deep sequencing, and their practical use in the clinical laboratory. Mycopathologia. 2017. https://doi.org/10.1007/ s11046-017-0185-x.

19. Rougeron A, Giraud S, Alastruey-Izquierdo A, et al. Ecology of Scedosporium species: present knowledge and future research. Mycopathologia. 2017. https://doi.org/10.1007/ s11046-017-0200-2.

20. Babič MN, Zupančič J, Gunde-Cimerman N, de Hoog S, Zalar P. Ecology of the human opportunistic black Yeast Exophiala dermatitidis indicates preference for humanmade habitats. Mycopathologia. 2017. https://doi.org/10. 1007/s11046-017-0134-8.

21. Scheffold A, Schwarz C, Bacher P. Fungus-Specific CD4 T Cells as specific sensors for identification of pulmonary fungal infections. Mycopathologia. 2017. https://doi.org/10. 1007/s11046-017-0229-2.

22. Staerck C, Vandeputte P, Gastebois A, et al. Enzymatic mechanisms involved in evasion of fungi to the oxidative stress: focus on Scedosporium apiospermum. Mycopathologia. 2017. https://doi.org/10.1007/s11046-0170160-6.

23. Pateau V, Razafimandimby B, Vandeputte P, et al. Gene disruption in Scedosporium aurantiacum: proof of concept with the disruption of SODC gene encoding a cytosolic $\mathrm{Cu}$. $\mathrm{Zn}$-superoxide dismutase. Mycopathologia. 2017. https:// doi.org/10.1007/s11046-017-0204-y.

24. Chen SC-A, Patel S, Meyer W, et al. Pseudomonas aeruginosa inhibits the growth of Scedosporium and Lomentospora in vitro. Mycopathologia. 2017. https://doi.org/10. 1007/s11046-017-0140-x.

25. Anand R, Moss RB, Sass G, et al. Small colony variants of Pseudomonas aeruginosa display heterogeneity in inhibiting Aspergillus fumigatus biofilm. Mycopathologia. 2017. https://doi.org/10.1007/s11046-017-0186-9.

26. Ramirez-Garcia A, Pellon A, Buldain I, et al. Proteomics as a tool to identify new targets against Aspergillus and Scedosporium in the context of cystic fibrosis. Mycopathologia. 2017. https://doi.org/10.1007/s11046-017-0139-3.

27. Cuénot S, Bouchara J-P. Atomic Force Microscopy: a promising tool for deciphering the pathogenic mechanisms of fungi in cystic fibrosis. Mycopathologia. 2017. https:// doi.org/10.1007/s11046-017-0201-1. 